\title{
Has the survival of patients with glioblastoma changed over the years?
}

R M deSouza, H Shaweis, C Han, V Sivasubramaniam, L Brazil, R Beaney, G Sadler, S Al-Sarraj, T Hampton, J Logan, V Hurwitz, R Bhangoo, R Gullan and K Ashkan

Correction to: British Journal of Cancer (2015) 114, 146-150. doi:10.1038/bjc.2015.421; published online 15 December 2015

Upon publication of the above paper in the British Journal of Cancer, one of the authors identified an error in the spelling of their name. V Sivasubramaniam appears correctly in the author list above. 